\title{
A study on the effect of the polymeric additive HPMC on morphology and
}

\section{polymorphism of ortho-aminobenzoic acid crystals}

\author{
E. Simone ${ }^{1}$, M.V. Cenzato ${ }^{2}$, Z.K. Nagy ${ }^{1,3, *}$ \\ ${ }^{1}$ Department of Chemical Engineering, Loughborough University, Loughborough, LE11 3TU, \\ UK; \\ ${ }^{2}$ Department of Chemical, Materials and Industrial Production Engineering (DICMAPI), \\ Università di Napoli Federico II, P.le Tecchio 80, 80125, Napoli, Italy \\ ${ }^{3}$ School of Chemical Engineering, Purdue University, West Lafayette, IN 47907-2100, USA \\ *Corresponding author. Email: zknagy@purdue.edu
}

\begin{abstract}
In the present study, the effect of Hydroxy Propyl Methyl Cellulose (HPMC) on the crystallization of ortho-aminobenzoic acid (OABA) was investigated by seeded and unseeded cooling crystallization experiments. The influence of HPMC on the induction time, crystal shape of Forms I and II of OABA and the polymorphic transformation time was studied. Furthermore, the capability of HPMC to inhibit growth of Form I was evaluated quantitatively and modelled using population balance equations (PBE) solved with the method of moments. The additive was found to strongly inhibit nucleation and growth of Form I as well as to increase the time for the polymorphic transformation from Form II to I. Solvent was also found to influence the shape of Form I crystals at equal concentrations of HPMC. In situ process analytical technology (PAT) tools, including Raman spectroscopy, focused beam reflectance measurement (FBRM) and attenuated total reflectance (ATR) UV-
\end{abstract}


Vis spectroscopy were used in combination with off-line techniques, such as optical microscopy, scanning electron microscopy (SEM), Raman spectroscopy, Malvern Mastersizer and differential scanning calorimetry (DSC) to study the crystals produced. The results illustrate how shape, size and stability of the two polymorphs of OABA can be controlled and tailored using a polymeric additive.

Keywords: A1.Crystal morphology; A1.Impurities; A2.Growth from solutions; B1.Organic compounds.

\section{Introduction}

Impurities in a crystallizer can have an incredibly large effect on the properties of the crystals produced in term of size distribution, shape and aspect ratio, polymorphic form and purity. They can inhibit crystal growth and delay nucleation as well as induce the nucleation of a different polymorphic form. A common model to describe the rate of crystal growth in solution in the presence of impurities was proposed in the late nineties by Kubota and Mullin [1][4]. The equations are based on the pinning mechanism of Cabrera and Vermilyea for the inhibition of step advancement considering one-dimensional adsorption of the impurities on the step lines. The model has been successfully used to quantitatively describe the growth rate of organic and inorganic compounds in the presence of impurities [4][10]. While growth kinetic in the presence of impurities is a relatively well-understood phenomenon [11]-[12] the effect on nucleation is still being studied [13]-[15]. Impurities can induce nucleation of a different polymorphic form [16]-[18] and have an effect on the time of polymorphic transformation [19]. The nucleation of the stable polymorph can be strongly inhibited by the presence of the impurity allowing the formation and growth of a metastable 
form [19]-[21]. Tailor-made and structurally related additives can promote nucleation of certain polymorphs because of structural similarities between them [22][23]. Additives can be absorbed on surfaces (templates) and induce heterogeneous nucleation of the target polymorph without the risk of incorporation in the crystal structure [24][29]. They can also delay nucleation [30] and promote the formation of very small crystals [31], sometimes in an amorphous form [14]. More often, polymeric additives (HPMC, PEG, PVP, PAA etc.), most of which are commonly used as excipients, have been used to modify nucleation and growth rates of several drugs. The use of common excipients to control polymorphism, shape and size of crystals is particularly convenient as they do not need to be removed or washed away after filtration. Vetter et al. (2011) used the surfactant Pluronic F127 to slow down the growth of ibuprofen [32]. Similar studies were conducted by Alonzo et al. (2012) and Oucherif et al. (2013) who analysed and modelled the growth and nucleation kinetics of felopidine in the presence of HPMC [33], [34]. The same research group also used HPMC to nucleate amorphous flutamide [14] and several other polymeric additives to inhibit growth of ritonavir [35-37]. In some cases polymers can promote growth: in particular, it was found that a very small amount of ethylene dinitrotetraacetic acid disodium salt (EDTA) could accelerate the growth of potassium dihydrogen phosphate (KDP) crystals by forming molecular complexes with adventitious impurities in solution and eliminating their inhibiting effect [38]. Recently, a mathematical description of the effect of one or more additive on the shape of KDP crystals was developed using population balance equations with the purpose of controlling the shape of the produced crystals [6][7].

This work constitutes a comprehensive study of the effect of the polymeric additive HPMC on the growth and nucleation mechanisms of two polymorphs of ortho-aminobenzoic acid. A combination of PAT tools and off-line solid state characterization techniques was used to understand the action of HPMC on the shape and size of the crystals of both Form I and II of 
the studied compound. The results of this study show how common excipients can be used to tailor morphology and polymorphism of organic compounds with significant impact on the manufacturing of pharmaceuticals in particular.

\section{Materials and methods}

Ortho-aminobenzoic acid (OABA) was purchased from Sigma-Aldrich (purity >98 \%). Isopropyl alcohol (IPA 99.97 \% grade, Fisher Scientific), and ultrapure water obtained via a Millipore ultra-pure water system were used as solvents. OABA has three known different polymorphic forms: (i) Form I is the stable form at room temperature, (ii) Form II is the most metastable at low temperature and it is usually obtained by cooling crystallization; it is enantiotropically related to Form I (transition temperature around $60{ }^{\circ} \mathrm{C}$ ) and monotropically related to the more stable Form III; (iii) Form III it is usually very difficult to nucleate during cooling crystallization from solution, and is normally produced by sublimation or by polymorphic transformation of Form I at high temperatures. Forms I and III are enantiotropically related with a transition temperature around $50{ }^{\circ} \mathrm{C}$ [39]-[41]. The solubility

of Form I and II in water and $10 \% \mathrm{w} / \mathrm{w}$ IPA and pure water were measured with the Avantium Crystal16 which uses turbidity measurement to detect the dissolution and nucleation points of a solution during temperature cycling. The measured solubility of Form I in pure water is:

$S_{I, w}=5.11 \cdot 10^{-7} e^{0.031 T}$

while the solubility of the same polymorph in $10 \% \mathrm{w} / \mathrm{w}$ IPA and water can be expressed as:

$S_{I, 10 \% I P A}=7.70 \cdot 10^{-10} e^{0.053 T}$

Form II solubility in pure water and water and $10 \%$ w/w IPA were measured as: 
$S_{I I, w}=9.08 \cdot 10^{-8} e^{0.037 T}$

$S_{I I, 10 \% I P A}=1.56 \cdot 10^{-9} e^{0.051 T}$

where $T$ is the temperature expressed in $\mathrm{K}$ and $S$ is the solubility in $\mathrm{g} / \mathrm{g}$ solvent.

An RXN2 Hybrid Raman analyser with immersion probe and 785 nm laser (Kaiser with iC Raman 4.1 software) was used, together with a D600L Lasentec FBRM probe (with FBRM software V 6.7.0), an MSC651 Carl Zeiss UV spectrophotometer with Hellma ATR (type 661.820-UV) probe (in-house LabView software). A $400 \mathrm{~mL}$ stirred and jacketed vessel fitted with a retreat curve impeller was used for the experiments. The temperature was controlled using a PT-100 temperature probe connected to a Huber Ministat 230 thermoregulator. The data from the FBRM, ATR-UV/Vis and the Huber were transmitted in real-time to the CryPRINS software (Crystallization Process Informatics System). This allows real-time monitoring of the FBRM counts, ATR-UV/Vis signal and the temperature, as well as setting a temperature profile and performing supersaturation control. The schematic of the rig is shown in Figure 1. Off-line analysis of the nucleated crystals was performed using a DXR 780nm Raman microscope (Thermo Scientific equipped with OMNIC 8 software).

\section{Methodology}

\subsection{Nucleation experiments for polymorphs I and II of OABA}

The aim of this set of experiments was to investigate the effect of HPMC on the kinetics of nucleation of OABA Forms I and II. In order to nucleate Form I, four aqueous solutions at $0.0097 \mathrm{~g} \mathrm{OABA} / \mathrm{g}$ water (saturation temperature around $49{ }^{\circ} \mathrm{C}$ ) were prepared and HMPC was then added to reach concentrations of $0,5,10,15 \mathrm{ppm}$. The temperature was raised to 
$50{ }^{\circ} \mathrm{C}$, kept constant for $30-40 \mathrm{~min}$ and then decreased at a cooling rate of $-1{ }^{\circ} \mathrm{C} / \mathrm{min}$ to $10{ }^{\circ} \mathrm{C}$, for each solution. The complete dissolution of the solids at high temperature as well as the nucleation temperature were detected by the FBRM, while the polymorphic outcome was identified by the online Raman, monitoring a peak at $770 \mathrm{~cm}^{-1}$ typical of Form II, and peaks at $1038 \mathrm{~cm}^{-1}$ and at $800 \mathrm{~cm}^{-1}$ for Form I. The whole temperature profile was repeated three times for each solution in order to check the consistency of the nucleation temperature detected. A $5 \mathrm{~mL}$ sample was taken after nucleation to confirm the polymorphic form nucleated off-line with the Raman microscope and DSC and to analyse the morphology of the crystals with an optical microscope. Further images were taken using a Hitachi TM3030 table top scanning electron microscope (SEM). Sputter coating consisted in $80 \%$ gold and $20 \%$ palladium and was realized applying $2.5 \mathrm{kV}$ for about 2 min in an argon atmosphere.

The analysis of the nucleation kinetics of Form II of OABA was carried out in solutions of $10 \%$ IPA and $90 \%$ water $\mathrm{w} / \mathrm{w}$ at $0.0133 \mathrm{~g} / \mathrm{g}$ solvent OABA concentration (corresponding to a saturation temperature of around $40{ }^{\circ} \mathrm{C}$ in respect to Form I). The amount of HPMC used was 0, 3, 5, 10 and 15 ppm and a new solution was prepared for each concentration of the additive. The procedure used was identical to the one described in the previous section, samples were taken and analysed off-line.

\subsection{Transformation experiments}

The effect of several concentrations of HPMC on the kinetics of the polymorphic transformation from Form II to Form I of OABA was investigated with this set of experiments.

Four solutions of $10 \%$ IPA and $90 \%$ water w/w at OABA concentration of $0.0133 \mathrm{~g} / \mathrm{g}$ solvent were prepared (saturation temperature around $40{ }^{\circ} \mathrm{C}$ ). HPMC was added in different concentrations: $0,3,5$ and $10 \mathrm{ppm}$. After the solution was prepared, the temperature was 
raised to $50{ }^{\circ} \mathrm{C}$ and kept for 30-40 min to allow a complete dissolution of the solid. After that, the temperature was decreased to $20{ }^{\circ} \mathrm{C}$ with a $-0.5^{\circ} \mathrm{C} /$ min cooling rate allowing nucleation of OABA Form II, and then kept constant at $20^{\circ} \mathrm{C}$ until complete transformation to Form I. Each transformation experiment was repeated at least twice using a new solution. The polymorphic transformation times were determined by tracking two specific Raman peaks corresponding to Forms I and II of OABA, respectively. Form I was associated to the 1038 $\mathrm{cm}^{-1}$ peak while Form II to the $770 \mathrm{~cm}^{-1}$ peak. The height to zero of the peaks was monitored during the experiment. After nucleation, $5 \mathrm{~mL}$ samples were taken during the polymorphic transformation and at the end of the experiment. The solids were analysed off-line to check the morphology and confirm the polymorphic composition.

\subsection{Form I growth experiments}

Isothermal seeded growth experiments were performed in water at additive concentrations of $0,0.1,0.75,5$ and $10 \mathrm{ppm}$. The seeds were obtained by cooling crystallization of OABA Form I in water (polymorphic outcome checked by off-line Raman analysis and DSC). Subsequently, metallic sieves were used to produce different size fractions; in particular, the size fraction chosen for these seeded growth experiments was 53-75 $\mu \mathrm{m}$.

The solutions were prepared adding solid OABA and HPMC to water in order to obtain a saturation temperature of $30{ }^{\circ} \mathrm{C}$ (around $0.0055 \mathrm{~g} \mathrm{OABA} / \mathrm{g}$ solvent). The temperature was raised and held at $40{ }^{\circ} \mathrm{C}$ for $20 \mathrm{~min}$ and then dropped to $20^{\circ} \mathrm{C}$. The seeds were added after the solution reached the temperature of $20^{\circ} \mathrm{C}$. The total amount of seeds was about $10 \%$ of the OABA concentration in solution in order to promote growth and avoid secondary nucleation. The seeds were left growing for about 15 hours after seeding. FBRM was used to check the complete dissolution of the solids in the supersaturated solution and the absence of secondary nucleation after seeding, while an ATR-UV/Vis probe was used to monitor the 
concentration of OABA in solution. Simple linear calibration functions (one for each different HPMC concentration) were determined using the height of the second derivative ATR-UV/Vis peak at $365 \mathrm{~nm}$ to measure the OABA solute concentration during the seeded experiments. At the end of each experiment, the solution was filtered and the crystals obtained were dried. A Malvern Mastersizer 2000 (with a Hydro 2000SM dispersant unit) allowed the determination of the crystal size distribution of both the seeds and the crystals at the end of the experiment. A saturated aqueous solution of OABA at ambient temperature was used as dispersant for the Mastersizer.

The growth kinetic constants at $20^{\circ} \mathrm{C}$ for each experiment at different HPMC concentrations were estimated using the data from the ATR-UV/Vis (solute concentration of OABA) and the initial crystal size distribution of the seeds measured with the Mastersizer.

A population balance model (PBM) based parameter estimation approach was used to determine the kinetic parameters for the system. For a seeded experiment in a batch stirred reactor, neglecting secondary nucleation, agglomeration and breakage, the population balance equation (PBE) can be written as:

$\frac{\partial f}{\partial t}+\frac{\partial(G f)}{\partial L}=0$

where $f$ is the average particle number density function, $t$ is time, $L$ is the particle characteristic length and $G$ is the growth rate which can be defined as:

$G=K_{g}(S-1)^{a}$

where $K_{g}$ is the growth kinetic constant (function of the temperature), $S$ is the supersaturation level defined as the ratio between solute concentration and equilibrium concentration at the considered temperature, and $a$ is the order of the growth kinetics.

In this work the method of moments was used to solve the PBE. Moments can be defined as a function of $f$ in the following way: 
$\mu_{\mathrm{j}}(\mathrm{t})=\int_{0}^{\infty} \mathrm{L}^{\mathrm{j}} f(\mathrm{~L}, \mathrm{t}) \mathrm{dL}$

Using the moments, equation (5) can be converted to a system of ordinary differential equations which can be easily solved. The first three moments (zeroth, first and second) were used for the parameter estimation:

$\frac{d \mu_{0}}{d t}=0$

$\frac{d \mu_{1}}{d t}=G \mu_{0}$

$\frac{d \mu_{2}}{d t}=2 G \mu_{1}$

The mass balance equation on OABA is written as:

$\frac{d C_{l i q}}{d t}=-3 k_{v} \rho_{c} G \mu_{2}$

where $C_{\text {liq }}$ is the solute concentration, $k_{v}$ is the shape factor ( $\pi / 6$ for spherical particles) and $\rho_{c}$ is the density of the crystals.

The parameters $K_{g}$ and $a$ will be estimated iteratively for all performed experiments by minimizing the following objective function:

Objective function $=\sum\left(C_{\text {sim }}-C_{\text {exp }}\right)^{2}$

The function ode15s in Matlab R2013a was used to solve the system of equations (8)-(11) and a combination of fminsearch and fmincom optimization factions was used to estimate $K_{g}$ and $a$ by minimizing the objective function (12). 


\section{Results and discussion}

\subsection{Nucleation of Form I (water)}

A lower nucleation temperature was recorded with increasing concentration of HPMC as shown in Figure 2. The addition of $10 \mathrm{ppm}$ of additive to the solvent generated a decrease in the nucleation temperature of more than $7^{\circ} \mathrm{C}$, which indicates that HPMC has an inhibitory effect on the nucleation of OABA Form I. It was also found that this additive can modify the shape of the crystals; therefore, it also inhibits growth of the stable polymorph. Figure 3 shows SEM images of crystals of Form I nucleated at different concentrations of HPMC. The shape changes from large plates at $0 \mathrm{ppm}$ of HPMC to long irregular needles at $15 \mathrm{ppm}$ of additive. A further experiment was performed nucleating Form I from water at very high concentration of HPMC (around $50 \mathrm{ppm}$ ) in order to investigate the peculiar shape obtained at $15 \mathrm{ppm}$ and study it in more detail using SEM. Images of the crystals obtained at $50 \mathrm{ppm}$ are shown in Figure 4. The presence of several steps of which growth has been stopped by the additive is clearly visible. Since HPMC is characterized by long polymeric molecules with multiple functional groups, even in low concentration it can have a strong inhibitory effect on the growth of OABA. In fact, the same polymeric chain can form multiple hydrogen bonds with many OABA molecules on several surfaces of the crystals and inhibit further growth of those faces. The result of this type of inhibition is the production of irregular needles such as the ones shown in Figure 3d and Figure 4.

\subsection{Nucleation of form II (90\% water and $10 \%$ IPA w/w)}

Similar nucleation experiments at several concentrations of HPMC were performed in $90 \%$ water and $10 \%$ IPA in order to study the effect of the additive on the kinetics of nucleation of the metastable Form II. Figure 5 shows the nucleation temperature of Form II at increasing 
concentration of HPMC. Dissimilarly to the case of Form I, nucleation of Form II does not seem to be affected by the presence of the additive; the nucleation temperatures do not change significantly with the addition of HPMC. However, the additive has clearly an effect on the growth of Form II crystals as shown in the SEM images in Figure 6. Form II nucleated from $10 \%$ IPA and $90 \%$ water w/w usually has a flat needle shape (see Figure 6a). With the addition of HPMC the edges of the crystals become more irregular with a similar effect that was noticed on Form I.

\subsection{Polymorphic transformation kinetics of Form II OABA crystals into Form I}

Although the nucleation temperature of Form II is not affected by the presence of HPMC in solution, a longer transformation time from Form II to I was observed. Table 1 shows the times of transformation from Form II to I in $10 \%$ IPA and water solutions at several amounts of HPMC dissolved. The transformation was completed in around 400 minutes in the absence of HPMC, while it took at least three times longer with the addition of only 3 ppm of additive; during the longest experiment performed at this concentration the transformation was about seven times longer compared to $0 \mathrm{ppm}$ of HPMC. The average transformation time at 3, 5 and $10 \mathrm{ppm}$ is very similar and a larger variability compared to the absence of HPMC can be

noticed. While at $0 \mathrm{ppm}$ the average transformation time was $364+51$ minutes, with only 51 minutes of standard deviation, for the experiments carried out at $3 \mathrm{ppm}$ the average transformation time increases to 1789 min with a much larger standard deviation of 1110 minutes. The average transformation time slightly increases at 5 and $10 \mathrm{ppm}$ of HPMC while the standard deviation remains large, around $1100 \mathrm{~min}$.

Figure 7 shows the normalized trend of the Raman peak for Form II during the transformation experiments at several HPMC concentrations. The slower transformation time at higher HPMC concentrations is partly due to the inhibition effect that HPMC has on the 
nucleation and growth of Form I, as shown in the previous sections. Form I nucleates later and grows slower in the presence of HPMC and, therefore, generates a delay in the completion of the transformation from Form II to I. It is worth noticing that crystals of Form I obtained after complete transformation from Form II in $10 \%$ IPA and water presents a considerably different shape compared to crystals of the same polymorph grown from water from both seeded and unseeded experiments. Figure 8 shows SEM pictures of Form I obtained after polymorphic transformation at different amounts of HPMC, indicating that these crystals present a three dimensional prismatic shape, which differs greatly from the flat irregular needles obtained from water. At 0 ppm of HPMC crystals of Form I are flat prisms less elongated compared to the ones obtained from water. As the amount of HPMC in solution increases, the width of the crystals increases as shown in Figure 8 (b and c). In conclusion, the shape of Form I crystals seems to be affected by both the presence of HPMC in solution but also by the combination solvent used and additive amount.

\subsection{Growth kinetics OABA Form I (water)}

A change in shape of the crystals of Form I in the presence of HPMC was observed in both water and $10 \%$ IPA and water, indicating that this additive has an effect on the growth kinetics of the stable polymorph. In order to quantitatively study this effect, further seeded isothermal experiments were conducted and a population balance model was used to estimate the growth kinetics of Form I at different amounts of HPMC in solution. Seeds of Form I crystals were added to supersaturated solutions at $20{ }^{\circ} \mathrm{C}$ and left to grow for about 15 hrs. After that time the suspension was filtered, the crystals dried and then measured with the Malvern Mastersizer. The final crystal size distributions (CSD) from the Malvern Mastersizer for each different amount of HPMC in solution are shown in Figure 9 a. The CSD of the seeds used for all the experiments is also shown in the figure for comparison. It can be 
noticed that for 0.75 and $5 \mathrm{ppm}$ of HPMC the final CSD is very close to the one of the seeds indicating very small growth for those concentrations of additive (the distribution of the crystals obtained at $10 \mathrm{ppm}$ is not shown since no significant difference was noticed in comparison to the initial seeds). The growth inhibition seems to be exponentially related to the amount of HPMC as shown by the trend of the volume mean diameter in Figure 9b. The same figure shows the standard deviation of the final crystal size distribution as a function of the concentration of HPMC in solution; an increase in this value, compared to the initial distribution of the seeds, can be observed for low concentrations of HPMC (0 and $0.1 \mathrm{ppm}$ ). This trend is typical of size dependent growth systems that can't be described using the method of moments to solve the population balance equations [42]. However, as shown in Figure 10, the simulated concentrations at different amounts of HPMC in solution follow reasonably well the experimental values, meaning that the model used, although very simple, is sufficient to represent the studied system, especially at high concentrations of additive.

The FBRM probe was used during the growth experiments to ensure the absence of secondary nucleation, breakage and/or agglomeration. Figure 11 shows the trend of the total counts during the seeded experiments: after an initial sudden increase due to seeding the vessel the number of counts remained stable, indicating that significant agglomeration and secondary nucleation or breakage were not occurring.

The kinetic constant and growth order at $20{ }^{\circ} \mathrm{C}$ for different amounts of HPMC in solution were estimated using the solute concentration data from the seeded desupersaturation experiments and the initial CSD of the seeds measured with the Malvern Mastersizer. The results of the parameter estimation are shown in Table 2 and Figure 10. The value of $K_{g}$ decreases exponentially as the amount of HPMC increases. Additionally, also the order of the growth rate decreases with higher concentrations of additive. 
The simulated solute concentration profiles for the seeded, isothermal desupersaturation experiments at several HPMC concentrations are shown in Figure 12. The same initial conditions (solute concentration and seeds CSD) were used in the simulations for a better comparison of the effect of different amounts of additive. The solute concentration decreases slower and slower as the HPMC amount in solution increases as a result of the growth inhibition generated by the additive.

\section{Conclusions}

In the present work a complete study on the effect of HPMC on nucleation and growth of two polymorphs of OABA was conducted. It was found that the additive inhibits nucleation of Form I but not for Form II. Additionally, because of the nucleation inhibition for the stable Form I, also the transformation time from Form II to Form I was found to be longer in the presence of HPMC.

Changes in shape for both Forms I and II were observed in the presence of the additive suggesting an inhibition of the growth rate for both polymorphs. It is worth noticing that the final shape of Form I strongly depended not only on the amount of HPMC in solution but also on the solvent used. Form I crystals grown from water presented a flat needle shape with edges that became more and more irregular as the amount of HPMC increased. Crystals of Form I grown from 10\% IPA and water instead presented a flat prismatic shape in the absence of HPMC with increasing width as the additive was added to the solution.

Seeded isothermal desupersaturation experiments were also performed in water in order to quantitatively estimate the growth kinetics of Form I at different amount of HPMC. It was found that the additive exponentially inhibit growth of Form I in the chosen solvent in good accordance with experimental data. 
This work shows the effectiveness of a polymeric additive in tailoring both the morphology and the polymorphism of an organic compound. Polymers can be used to modify shape and size of the crystals in order to improve the properties of the final drug as well as the downstream operations. The main advantages of this type of additives are their intrinsic safety (many are already used as excipients in pharmaceutical formulations) and the decreased likelihood of significant incorporation in the API crystal structure due to their large molecular size, thus having significant effect on morphology and polymorphism often without detectable effect on purity.

\section{Acknowledgements}

Financial support provided by the European Research Council grant no. [280106-CrySys]. The second author acknowledges the University of Napoli Federico II for additional support.

\section{References}

[1] N. Kubota, Effect of impurities on the growth kinetics of crystals, Cryst. Res. Technol., 8 (2001) 749-769. http://onlinelibrary.wiley.com/doi/10.1002/15214079(200110)36:8/10\%3C749::AID-CRAT749\%3E3.0.CO;2-\%23/abstract

[2] N. Kubota, M. Yokota, J.W. Mullin, The combined influence of supersaturation and impurity concentration on crystal growth, J. Cryst. Growth, 212 (2000) 480-488. http://www.sciencedirect.com/science/article/pii/S0022024800003390

[3] N. Kubota, M. Yokota, J.W. Mullin, Supersaturation dependence of crystal growth in solutions in the presence of impurity, J.Cryst. Growth, 182 (1997) 86-94. http://www.sciencedirect.com/science/article/pii/S002202489700328X 
[4] N. Kubota, J.W. Mullin, A kinetic model for crystal growth from aqueous solution in the presence of impurity, J. Cryst. Growth, 152 (1995). 203-208. http://www.sciencedirect.com/science/article/pii/002202489500128X

[5] Majumder, A., Nagy, ZK. Prediction and Control of Crystal Shape Distribution in the Presence of Crystal Growth Modifiers, Chem. Eng. Sci., 101 (2013) 593-602. http://www.sciencedirect.com/science/article/pii/S0009250913005101

[6] A. Borsos, A. Majumder, Z.K. Nagy, Model Development and Experimental Validation for Crystal Shape Control by Using Tailored Mixtures of Crystal Growth Modifiers, Comput. Aided Chem. Eng., 33 (2014) 781-786. http://www.sciencedirect.com/science/article/pii/B9780444634566501319

[7] A. Borsos, A. Majumder, Z.K. Nagy, Multi-impurity adsorption model for modeling crystal purity and shape evolution during crystallization processes in impure media, Cryst. Growth Des., 16 (2016) 555-568. http://pubs.acs.org/doi/abs/10.1021/acs.cgd.5b00320

[8] U.S: Kestur, L.S. Taylor, Evaluation of the crystal growth rate of felodipine polymorphs in the presence and absence of additives as a function of temperature, Cryst. Growth Des., 13 (2913) 4349-4354. http://pubs.acs.org/doi/abs/10.1021/cg400708p

[9] W.J.P. van Enckevort, A.C.J.F. van der Berg, K.B.G. Kreuwel, A.J. Derksen, Impurity blocking of growth steps: experiments and theory, J. Crys. Growth, 166 (1996). 156-161. http://www.sciencedirect.com/science/article/pii/0022024896001364

[10] F. Fevotte, G. Fevotte, A method of characteristics for solving population balance equations (PBE) describing the adsorption of impurities during crystallization processes, Chem. Eng. Sci., 65 (2010) 3191-3198. http://www.sciencedirect.com/science/article/pii/S0009250910000758 
[11] E. Simone, W. Zhang, Z.K. Nagy, Analysis of the crystallization process of a biopharmaceutical compound in the presence of impurities using process analytical technology (PAT) tools, J Chem Technol Biotechnol 91 (2016) 1461-1470. http://onlinelibrary.wiley.com/doi/10.1002/jctb.4743/abstract

[12] E. Simone, W. Zhang, Z.K. Nagy, Application of Process Analytical TechnologyBased Feedback Control Strategies To Improve Purity and Size Distribution in Biopharmaceutical Crystallization, Cryst. Growth Des. 15 (2015) 2908-2919. http://pubs.acs.org/doi/abs/10.1021/acs.cgd.5b00337

[13] M. Salvalaglio, T. Vetter, F. Giberti, M. Mazzotti, M., Parrinello, Uncovering molecular details of urea crystal growth in the presence of additives, J. Am. Chem. Soc., 134 (2012) 17221-17233. http://pubs.acs.org/doi/abs/10.1021/ja307408x

[14] N.S. Trasi, L.S. Taylor, Effect of additives on the crystal growth and nucleation of amorphous flutamide, Cryst. Growth Des., 12 (2012) 3221-3230. http://pubs.acs.org/doi/abs/10.1021/cg300370q

[15] J. Anwar, P.K. Boateng, R. Tamaki, S. Odedra, Mode of action and design rules for additives that modulate crystal nucleation, Angew. Chem. Int. Ed., 48 (2009) 15961600. http://onlinelibrary.wiley.com/doi/10.1002/anie.200804553/full

[16] C.H. Gui, K. Chatterjee, V.J. Young, D.J.W. Grant, Stabilization of a metastable polymorph of sulfamerazine by structurally related additives, J.Cryst. Growth, 235 (2002). 471-481.

http://www.sciencedirect.com/science/article/pii/S0022024801017845

[17] X. He, J.G. Stowell, K.R. Morris, R.R. Pfeiffer, H. Li, G.P. Stahly, S.R. Byrn, Stabilization of a metastable polymorph of 4-methyl-2-nitroacetanilide by isomorphic additives, Cryst. Growth Des. , (2001) 305-312. http://pubs.acs.org/doi/abs/10.1021/cg0055225 
[18] S.K. Poornachary, P.S. Chow, R.B.H. Tan, Influence of solution speciation of impurities on polymorphic nucleation in glycine, Cryst. Growth Des. , 8 (2008) 179185. http://pubs.acs.org/doi/abs/10.1021/cg060570w

[19] Mukuta, T., Lee, A.Y., Kawakami, T., Myerson, A.S. 2005, Influence of impurities on the solution-mediated phase transformation of an active pharmaceutical ingredient, Cryst. Growth and Des., vol. 5, no. 4, pp. 1429-1436.

http://pubs.acs.org/doi/abs/10.1021/cg049646j

[20] Y. Mo, L. Dang, H. Wei, L-glutamic acid polymorph control using amino acid additives, Ind. Eng. Chem. Res., 50 (2011) 10385-10392. http://pubs.acs.org/doi/abs/10.1021/ie102152d

[21] A.N. Saleemi, I.I. Onyemelukwe, Z.K. Nagy, Effect of a structurally related substance on the crystallization of paracetamol, Front. Chem. Sci. Eng., 7 (2013) 79-87. http://link.springer.com/article/10.1007\%2Fs11705-013-1308-7\#page-1

[22] C.P. Price, A.L. Grzesiak, A.J. Matzger, Crystalline polymorph selection and discovery with polymer heteronuclei, J. Am. Chem. Soc., vol. 127 (2005) 5512-5517. http://pubs.acs.org/doi/abs/10.1021/ja042561m

[23] I. Weissbuch, R. Popovitz-Biro, M. Lahav, L. Leiserowitz, Understanding and control of nucleation, growth, habit, dissolution and structure of two- and three- dimensional crystals using "tailor-made" auxiliaries, Acta Crystallogr. B, B51 (1995) 115-148. http://scripts.iucr.org/cgi-bin/paper?pii=S0108768194012061\&sentby=wiley

[24] Y. Lang, A.M. Cervantes, L.T: Biegler, Dynamic optimization of a batch cooling crystallization process, Ind. Eng. Chem. Res., vol. 38 (1999) 1469-1477. http://pubs.acs.org/doi/abs/10.1021/ie980585u 
[25] S.A. Kulkarni, C.C: Weber, A.S. Myerson, J.H. ter Horst, Self-association during heterogeneous nucleation onto well-defined templates, Langmuir, 30 (2014) 1236812375. http://pubs.acs.org/doi/abs/10.1021/la5024828

[26] R. Hiremath, J.A. Basile, S.W. Varney, J.A. Swift, Controlling molecular crystal polymorphism with self assembled monolayer templates, J. Am. Chem. Soc., 127 (2005) 18321-18327. http://pubs.acs.org/doi/abs/10.1021/ja0565119

[27] G. Di Profio, E. Fontanova, E. Curcio, E. Drioli, From tailored supports to controlled nucleation: exploring material chemistry, surface nanostructure, and wetting regime effects in heterogeneous nucleation of organic molecules, Cryst. Growth Des., 12 (2912) 3749-3757. http://pubs.acs.org/doi/abs/10.1021/cg3005568

[28] K. Chadwick, A.S. Myerson, B Trout, Polymorphic control by heterogeneous nucleation- A new method for selecting crystalline substrates, CrystEngComm, 13 (2011) 6625.

http://pubs.rsc.org/en/content/articlelanding/ce/2011/c1ce05871a\#!divAbstract

[29] S.J. Bonafede, M.D. Ward, Selective nucleation and growth of an organic polymorph by ledge-directed epitaxy on a molecular crystal substrate, J. Am. Chem. Soc., 117 (1995) 7853-7861. http://pubs.acs.org/doi/abs/10.1021/ja00135a001

[30] G. Fevotte, N. Gherras, J. Moutte, Batch cooling solution crystallization of ammonium oxalate in the presence of impurities: study of solubility, supersaturation, and steady-state inhibition, Cryst. Growth Des., 13 (2013) 2737-2748. http://pubs.acs.org/doi/abs/10.1021/cg301737s

[31] N.M. Reis, Z.K. Liu, C.M. Reis, M.R. Mackley, Hydroxypropyl methylcellulose as a novel tool for isothermal solution crystallization of micronized paracetamol, Cryst.l Growth Des., 14 (2014) 3191-3198. http://pubs.acs.org/doi/abs/10.1021/cg4009637 
[32] T. Vetter, M. Mazzotti, J. Brozio, Slowing the growth rate of ibuprofen crystals using the polymeric additive pluronic F127, Cryst. Growth Des., 11 (2011) 3813-3821. http://pubs.acs.org/doi/abs/10.1021/cg200352u

[33] K.A. Oucherif, S. Raina, L.S. Taylor, J.D. Lister, Quantitative analysis of the inhibitory effect of HPMC on felodipine crystallization kinetics using population balance modeling, CrystEngComm, 15 (2013) 2197-2205. http://pubs.rsc.org/en/content/articlehtml/2013/ce/c2ce26490k

[34] D.E. Alonzo, S. Raina, D. Zhou, Y. Gao, G.G.Z. Zhang, L.S. Taylor, Characterizing the impact of hydroxypropylmethyl cellulose on the growth and nucleation kinetics of felodipine from supersaturated solutions, Cryst. Growth Des., vol. 12 (2012) 15381547. http://pubs.acs.org/doi/abs/10.1021/cg201590j

[35] G.A. Ilevbare, H. Liu, K.J. Edgar, L.S. Taylor, Effect of binary additive combinations on solution crystal growth of the poorly water-soluble drug, Ritonavir, Cryst. Growth Des., 12 (2012) 6050-6060. http://pubs.acs.org/doi/abs/10.1021/cg301169t

[36] G.A. Ilevbare, H. Liu, K.J. Edgar, L.S. Taylor, Inhibition of solution crystal growth of ritonavir by cellulose polymers - factors influencing polymer effectiveness, CrystEngComm, 14 (2012) 6503-6514. http://pubs.rsc.org/en/content/articlelanding/2012/ce/c2ce25515d\#!divAbstract

[37] G.A. Ilevbare, H. Liu, K.J. Edgar, L.S. Taylor, Understanding polymer properties important for crystal growth inhibition- impact of chemically diverse polymers on solution crystal growth of ritonavir, Cryst. Growth Des., 12 (2012) 3133-3143. http://pubs.acs.org/doi/abs/10.1021/cg300325p

[38] V.A. Kuznetsov, T.M. Okhrimenko, M. Rak, Growth promoting effect of organic impurities on growth kinetics of KAP and KDP crystals, J. Cryst.Growth, 193 (1998) 164-173. http://www.sciencedirect.com/science/article/pii/S0022024898004898 
[39] S. Jiang, J.H. ter Horst, P.J. Jansens, Control over polymorph formation of oaminobenzoic acid, Cryst.Growth Des., 10 (2010) 2541-2547. http://pubs.acs.org/doi/abs/10.1021/cg901257s

[40] S. Jiang, J.H. ter Horst, P.J. Jansens, Mechanism and kinetics of polymorphic transformation of o-aminobenzoic acid, Cryst. Growth Des., 10 (2010) 2123-2128. http://pubs.acs.org/doi/abs/10.1021/cg901256u

[41] S. Jiang, J.H. ter Horst, P.J. Jansens, Concomitant polymorphism of o-aminobenzoic acid in antisolvent crystallization, Cryst. Growth Des., 8 (2010) 37-43. http://pubs.acs.org/doi/abs/10.1021/cg070517n

[42] E. Aamir, Z. K. Nagy, C. D. Rielly, T. Kleinert, B. Judat, Combined quadrature method of moments and method of characteristics approach for efficient solution of population balance models for dynamic modeling and crystal size distribution control of crystallization processes, Ind. Eng. Chem. Res., 48 (2009) 8575-8584. http://pubs.acs.org/doi/abs/10.1021/ie900430t 


\section{List of Tables:}

Table 1: Polymorphic transformation times of OABA from Form II to I in the presence of HPMC in solution.

Table 2: Estimated growth kinetic parameters of OABA Form I crystals for the seeded

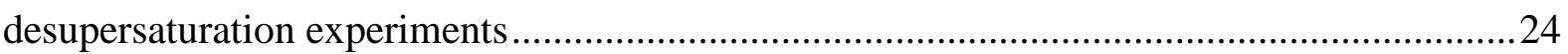


Table 1: Polymorphic transformation times of OABA from Form II to I in the presence of HPMC in solution.

\begin{tabular}{cc}
\hline $\begin{array}{c}\text { HPMC content } \\
\text { (ppm) }\end{array}$ & Transformation time (min) \\
\hline $\mathbf{0}$ & $364 \pm 51$ \\
\hline $\mathbf{3}$ & $1789 \pm 1110$ \\
\hline $\mathbf{5}$ & $1871 \pm 1076$ \\
\hline $\mathbf{1 0}$ & $1903 \pm 1141$ \\
\hline
\end{tabular}


Table 2: Estimated growth kinetic parameters of OABA Form I crystals for the seeded desupersaturation experiments

\begin{tabular}{ccccc}
\hline HPMC & $\ln \left(\boldsymbol{K}_{\boldsymbol{g}}\right)$ & $\boldsymbol{a}$ & $\boldsymbol{K}_{\boldsymbol{g}}$ & Objective function \\
$\mathbf{( p p m )}$ & & & & value \\
\hline 0 & -17.5 & 1.4 & $2.5 \mathrm{E}-08$ & $5.45 \mathrm{E}-05$ \\
\hline 0.1 & -18.3 & 1.1 & $1.2 \mathrm{E}-08$ & $2.31 \mathrm{E}-04$ \\
\hline 0.75 & -18.8 & 0.9 & $6.8 \mathrm{E}-09$ & $1.27 \mathrm{E}-04$ \\
\hline 5 & -19.6 & 0.6 & $3.1 \mathrm{E}-09$ & $4.43 \mathrm{E}-05$ \\
\hline 10 & & & & $1.14 \mathrm{E}-04$ \\
\hline
\end{tabular}




\section{List of Figures:}

Figure 1: Schematic of the rig used for the experiments indicating the Crystallization Process Informatics System (CryPRINS) and composite PAT array.

Figure 2: Nucleation temperature of Form $\mathrm{I}$ in water as a function of the concentration of HPMC. .28

Figure 3: SEM images of OABA Form I nucleated from water in the presence of different concentrations of HPMC (a) 0 ppm (b) 5 ppm (c) 10 ppm and (d) 15 ppm.

Figure 4: SEM images of crystals of OABA Form I grown in water in the presence of $50 \mathrm{ppm}$ of HPMC in solution. .30

Figure 5: Nucleation temperatures of Form II in water as a function of the concentration of HPMC .31

Figure 6: SEM images of OABA Form II nucleated in 10\% IPA and water solvent and at three different concentrations of HPMC (a) 0 ppm (b) 3 ppm (c) 15 ppm. 32

Figure 7: Polymorphic transformation times as a function of the concentration of HPMC measured using Raman spectroscopy. .33

Figure 8: SEM images of Form I crystals obtained after complete transformation of OABA Form II nucleated in $10 \%$ IPA and water solutions at different concentrations of HPMC (a) 0 ppm (b) 3 ppm and (c) 15 ppm.

Figure 9: (a) Final crystal size distribution of OABA form I crystals for seeded growth experiments at constant temperature and different HPMC concentrations; (b) Standard deviation and volume mean diameter $\mathrm{D}[4,3]$ of the distribution measured with the Malvern Mastersizer at different HPMC concentrations. .35

Figure 10: Total counts trends during the seeded growth experiments .37 
Figure 11: Experimental and simulated OABA solute concentration during the seeded growth experiments in the present of (a) 0 ppm (b) $0.1 \mathrm{ppm}$ (c) $0.75 \mathrm{ppm}$ (d) $5 \mathrm{ppm}$ and (e) $10 \mathrm{ppm}$

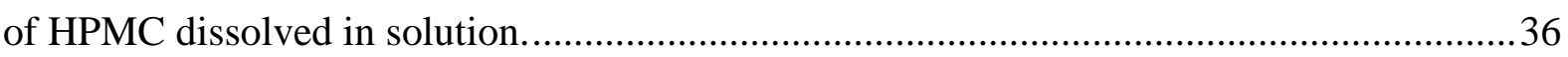

Figure 12: Simulated concentration profiles for seeded desupersaturation experiments of OABA at different concentrations of HPMC. .38 


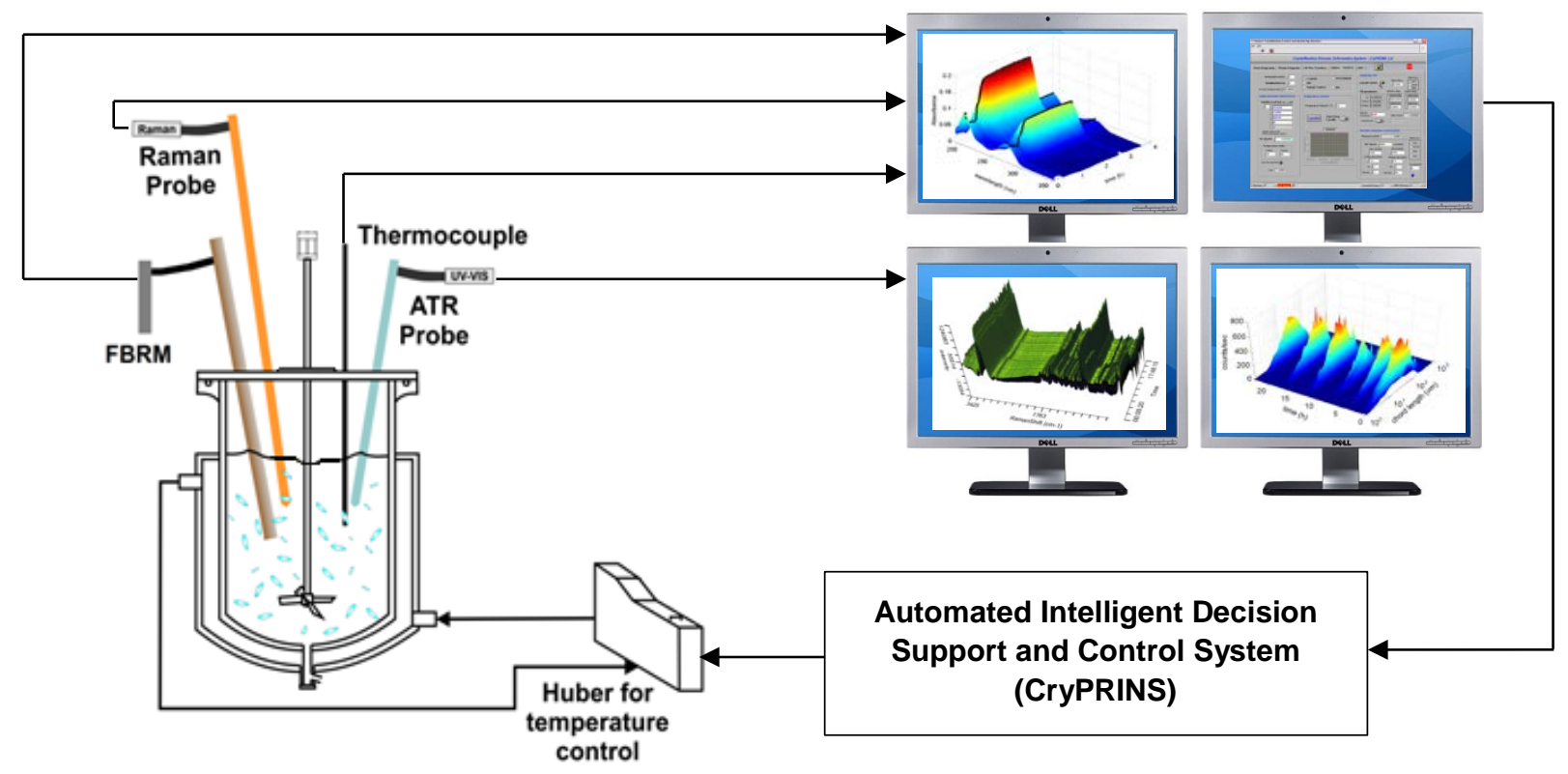

Figure 1: Schematic of the rig used for the experiments indicating the Crystallization Process Informatics System (CryPRINS) and composite PAT array. 


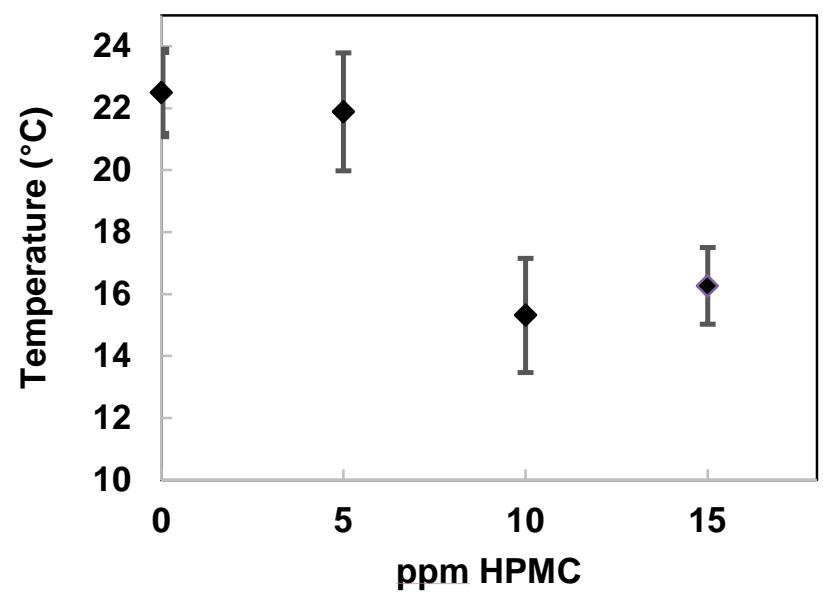

Figure 2: Nucleation temperature of Form $I$ in water as a function of the concentration of HPMC. 


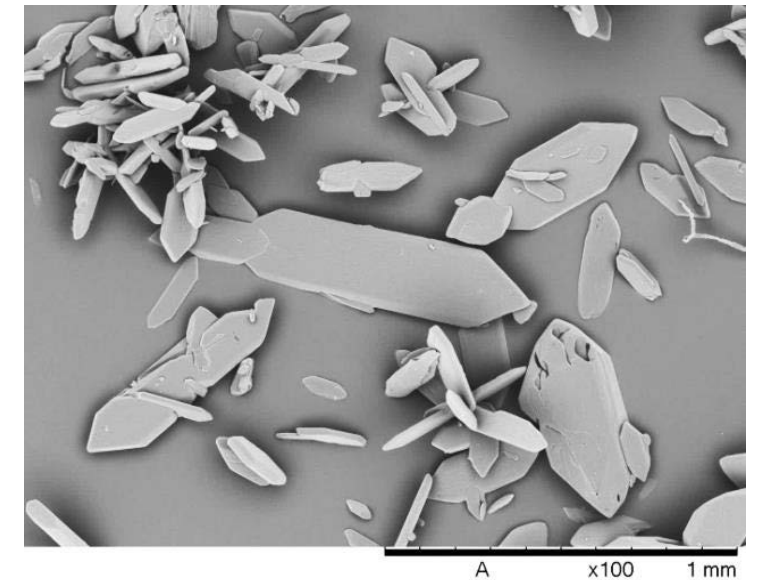

(a)

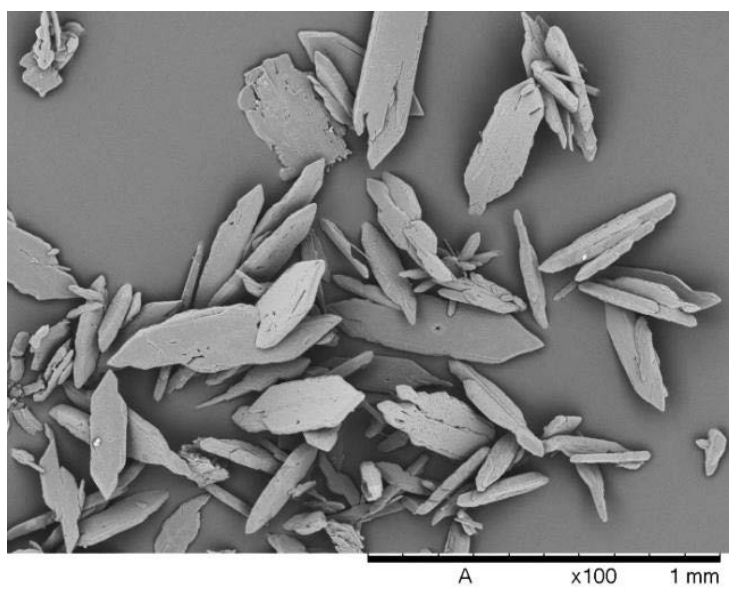

(c)

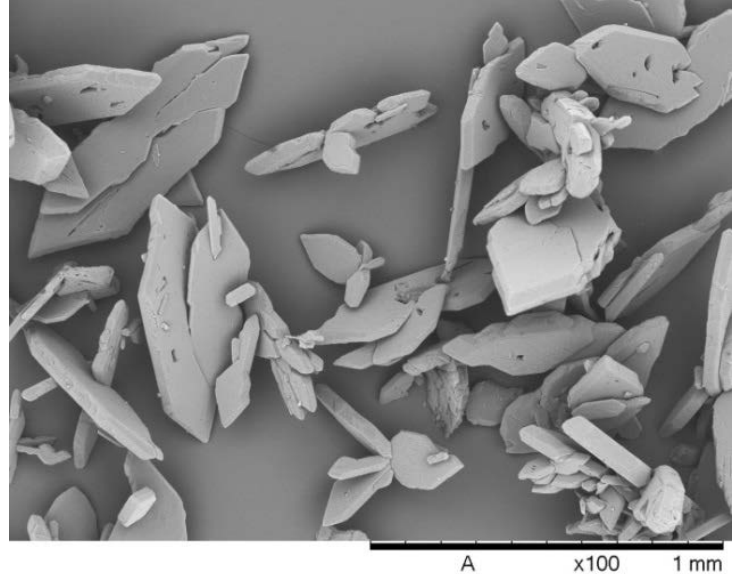

(b)

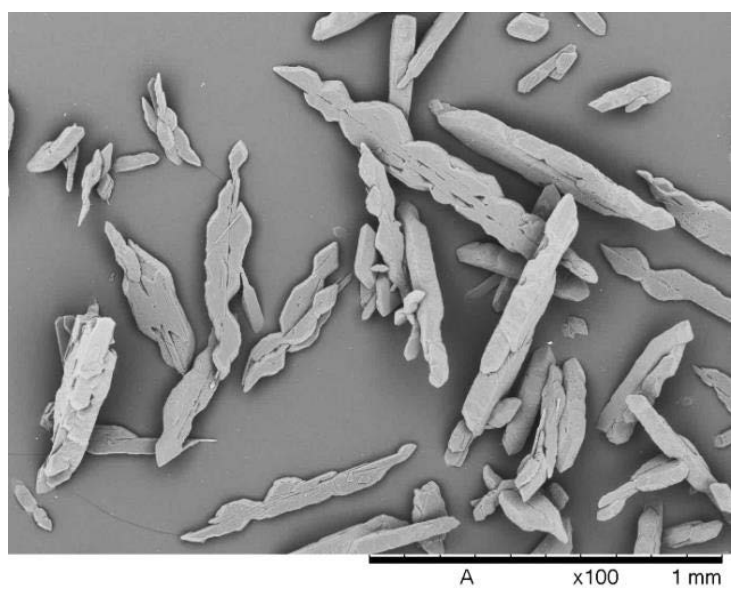

(d)

Figure 3: SEM images of OABA Form I nucleated from water in the presence of different concentrations of HPMC (a) 0 ppm (b) 5 ppm (c) 10 ppm and (d) 15 ppm. 

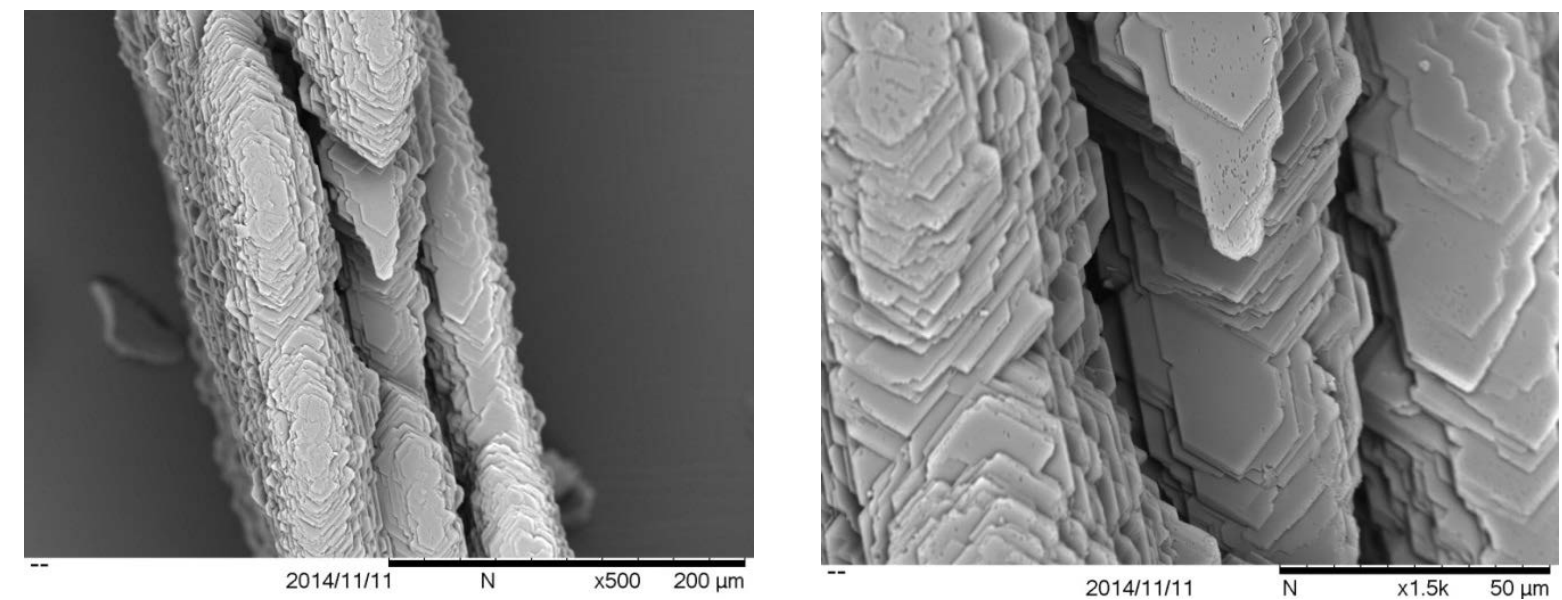

Figure 4: SEM images of crystals of OABA Form I grown in water in the presence of 50 ppm of HPMC in solution. 


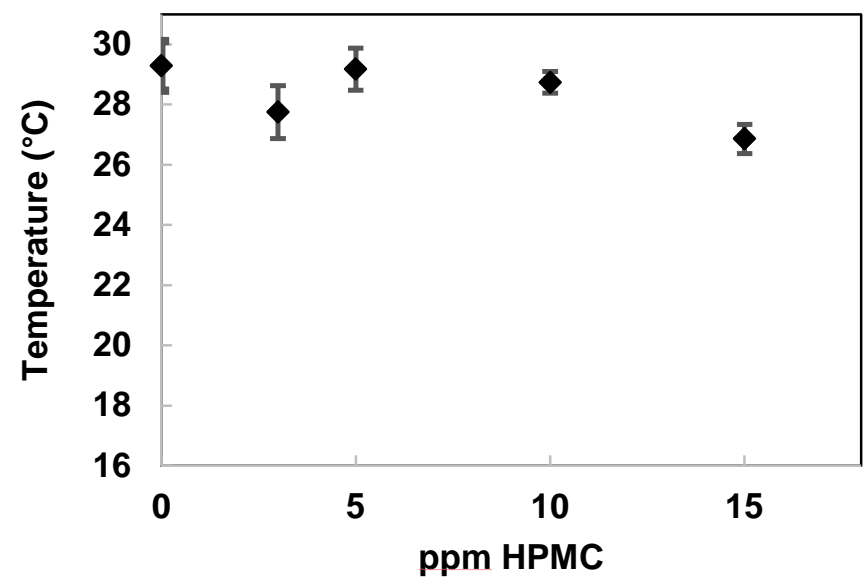

Figure 5: Nucleation temperatures of Form II in water as a function of the concentration of HPMC. 


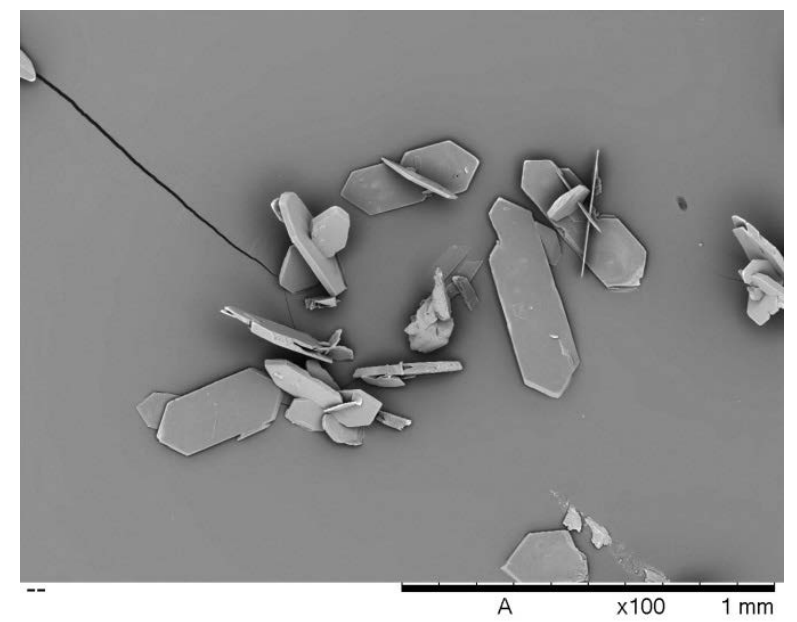

(a)

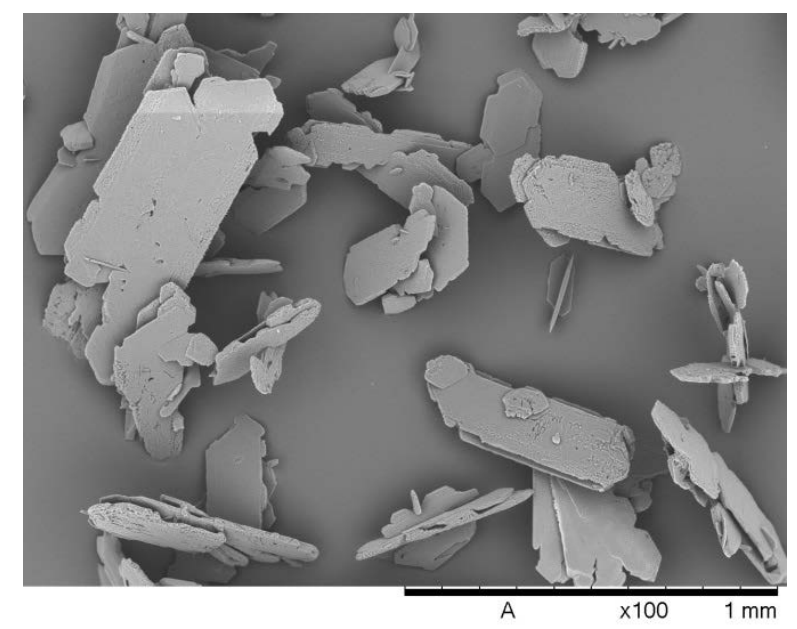

(b)

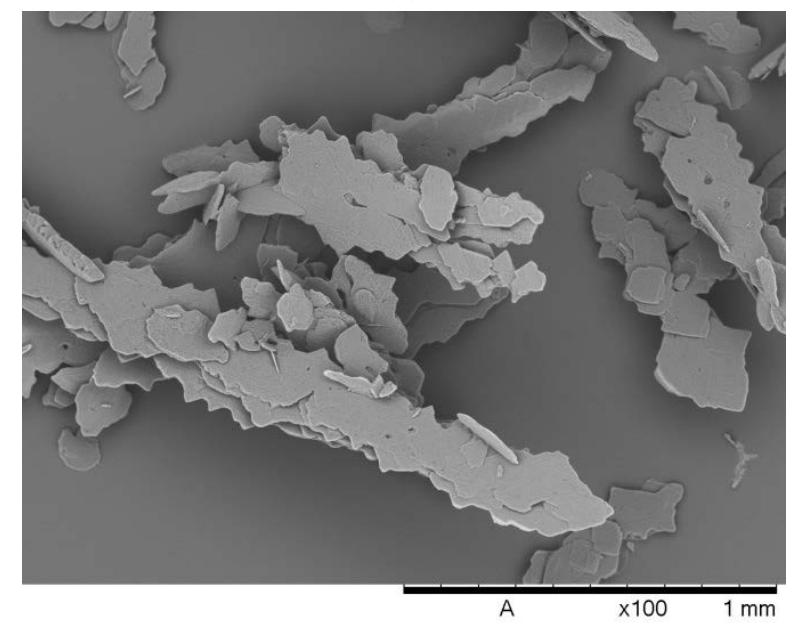

(c)

Figure 6: SEM images of OABA Form II nucleated in 10\% IPA and water solvent and at three different concentrations of HPMC (a) 0 ppm (b) 3 ppm (c) 15 ppm. 


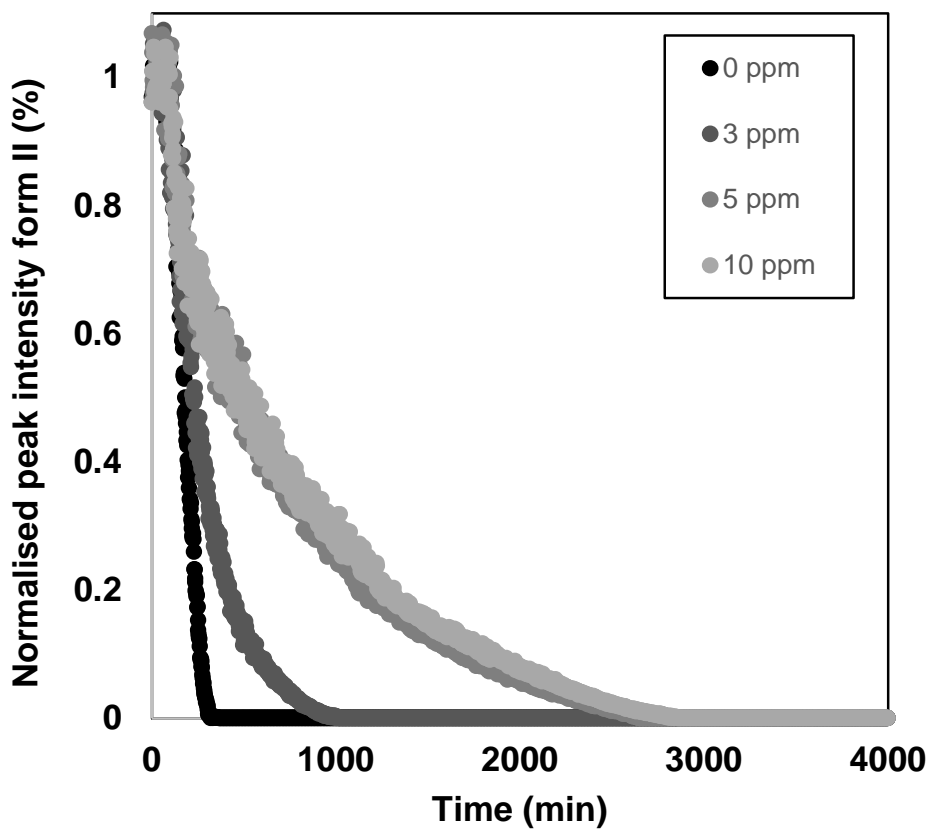

Figure 7: Polymorphic transformation times as a function of the concentration of HPMC measured using Raman spectroscopy. 


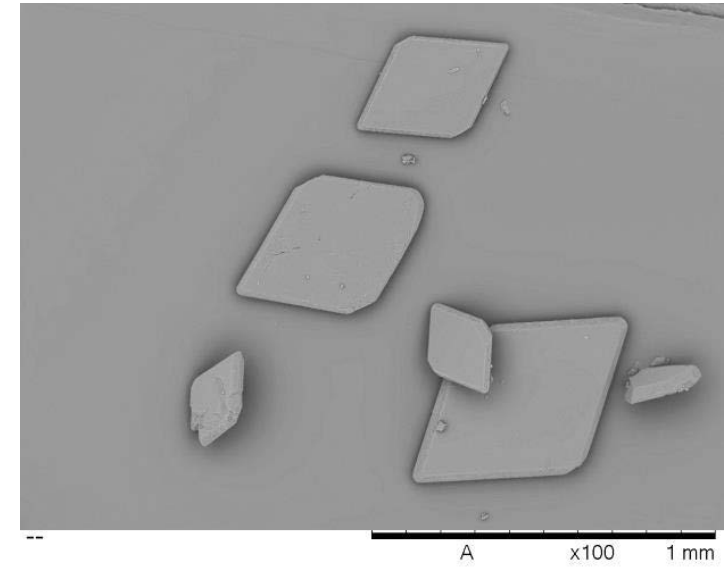

(a)

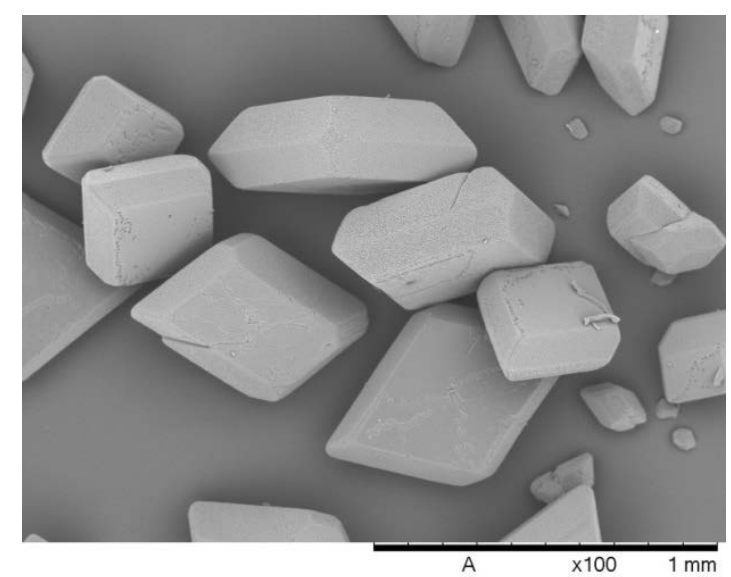

(b)

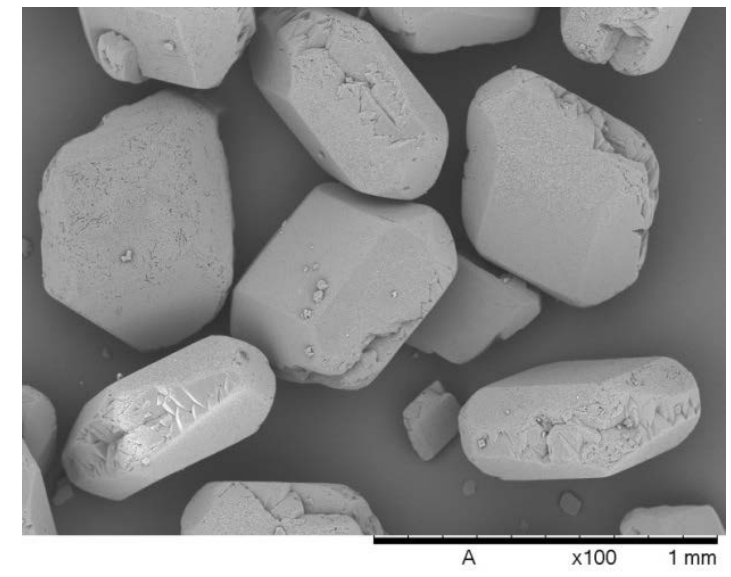

(c)

Figure 8: SEM images of Form I crystals obtained after complete transformation of OABA Form II nucleated in 10 \% IPA and water solutions at different concentrations of HPMC (a) 0 ppm (b) 3 ppm and (c) 15 ppm. 

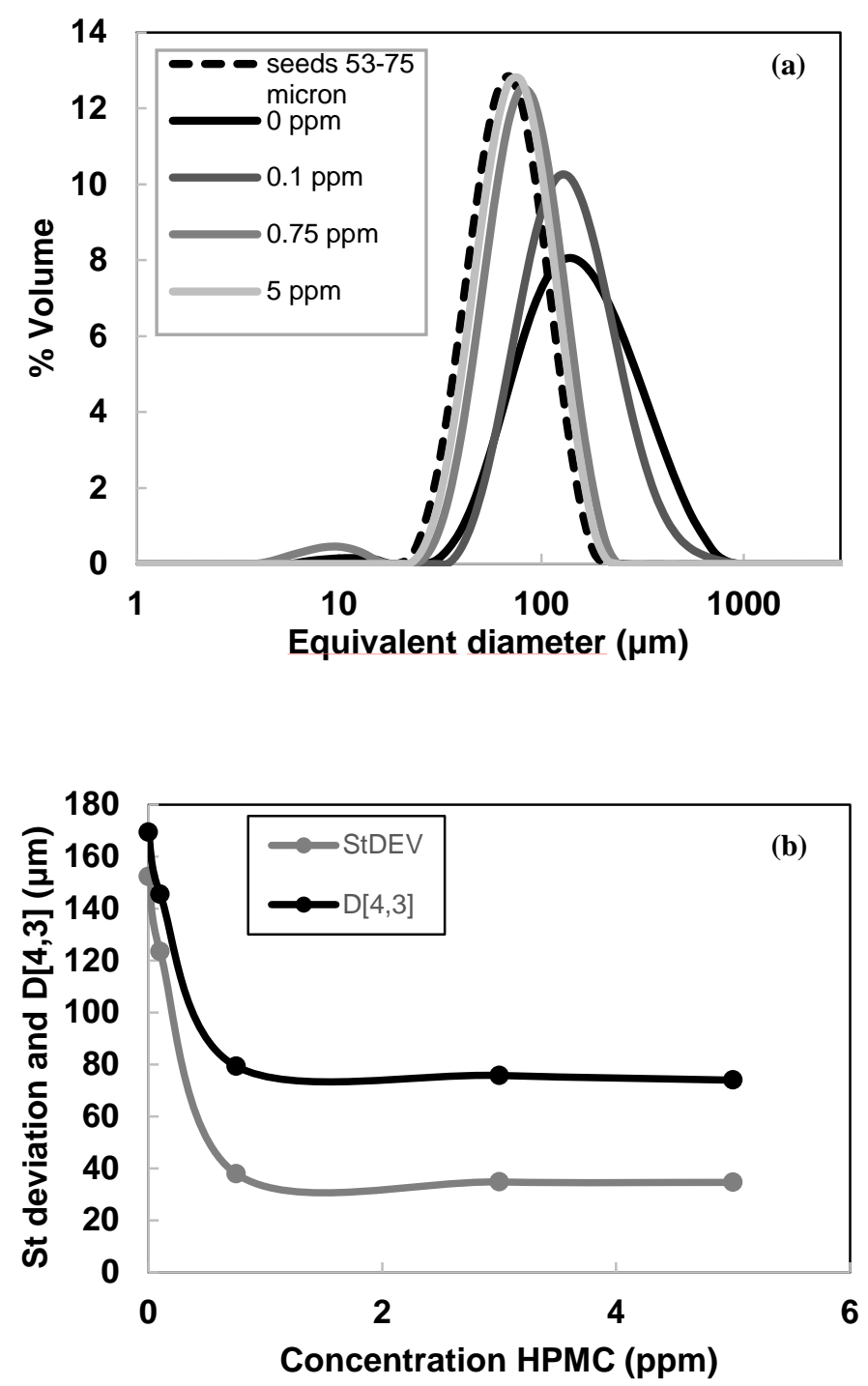

Figure 9: (a) Final crystal size distribution of OABA form I crystals for seeded growth experiments at constant temperature and different HPMC concentrations; (b) Standard deviation and volume mean diameter D[4,3] of the distribution measured with the Malvern Mastersizer at different HPMC concentrations. 


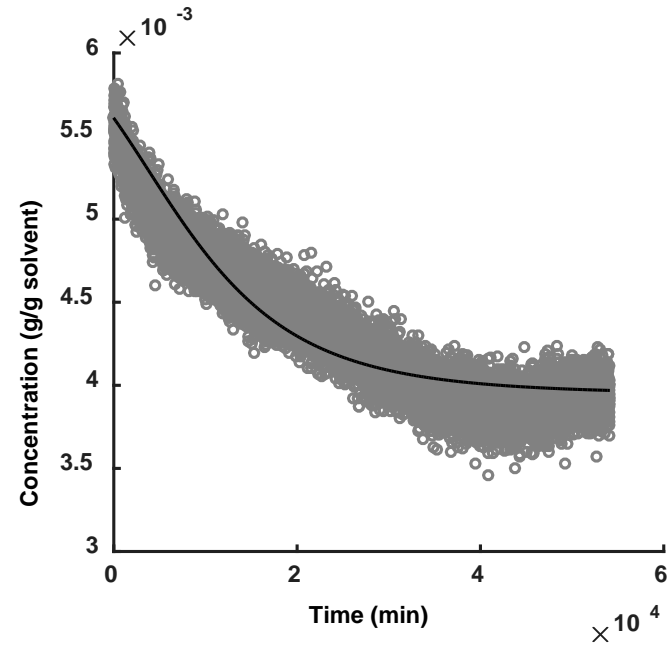

(a)

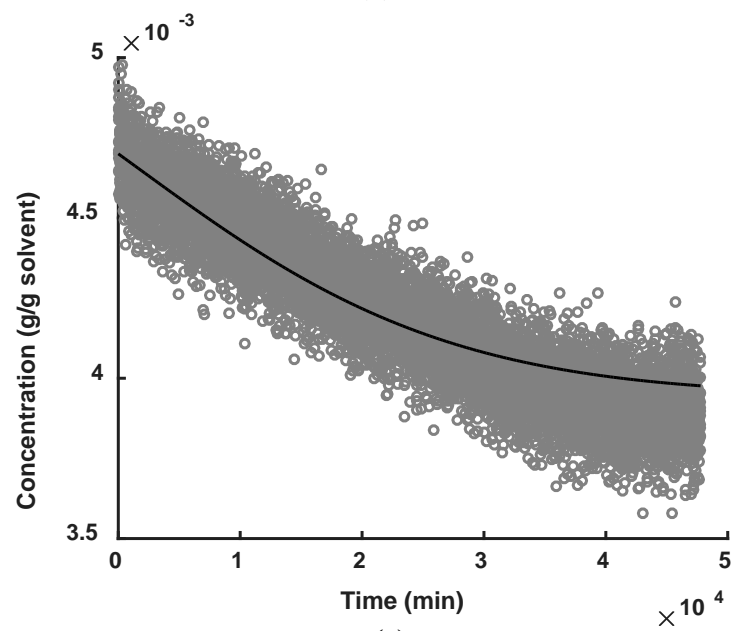

(c)

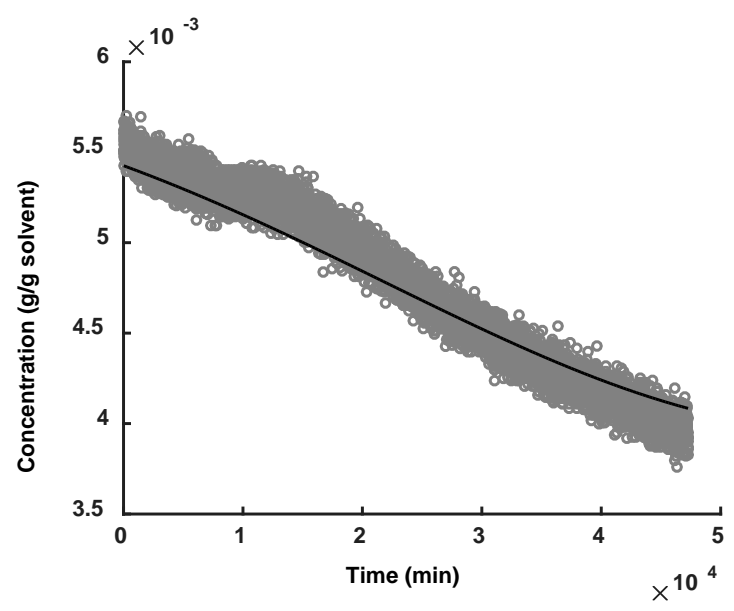

(e)

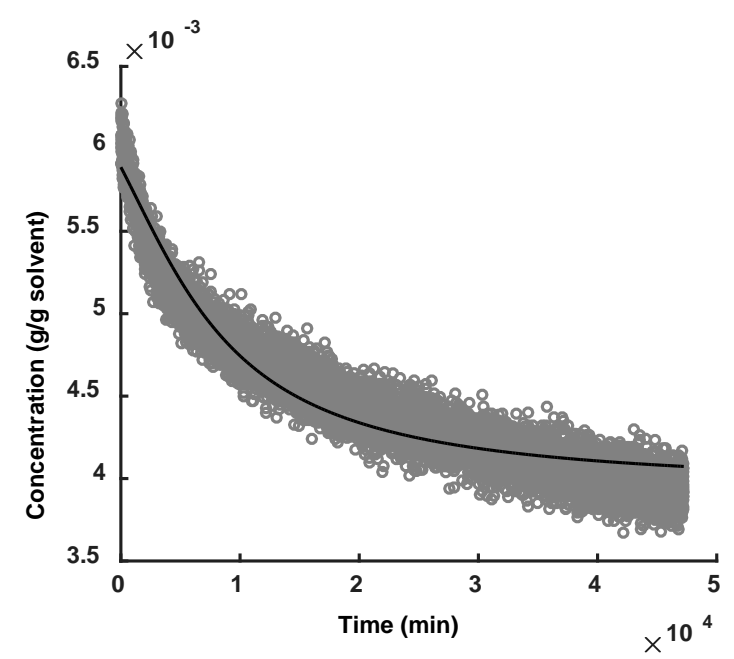

(b)

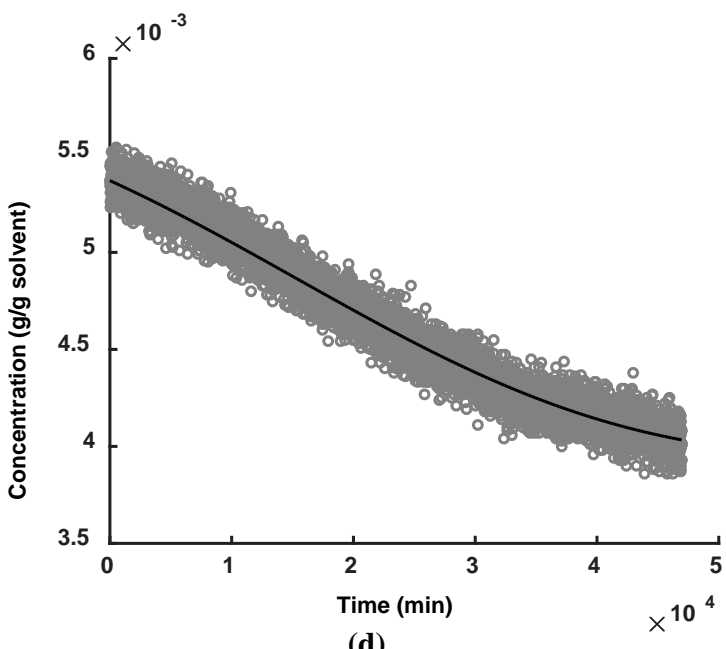

(d)

Figure 10: Experimental and simulated OABA solute concentration during the seeded growth experiments in the present of (a) 0 ppm (b) 0.1 ppm (c) 0.75 ppm (d) 5 ppm and (e) 10 ppm of HPMC dissolved in solution. 


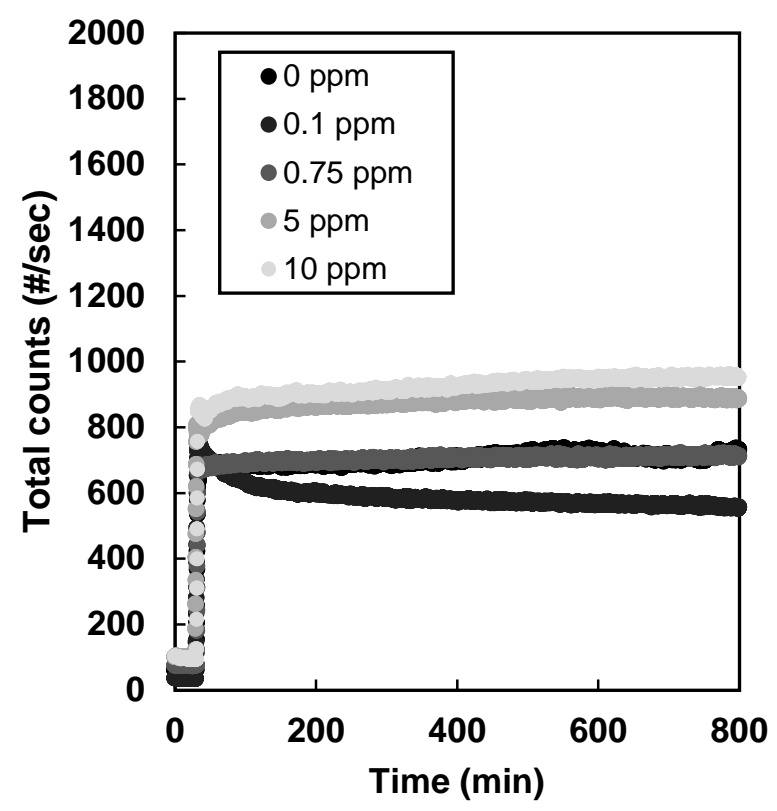

Figure 11: Total counts trends during the seeded growth experiments 


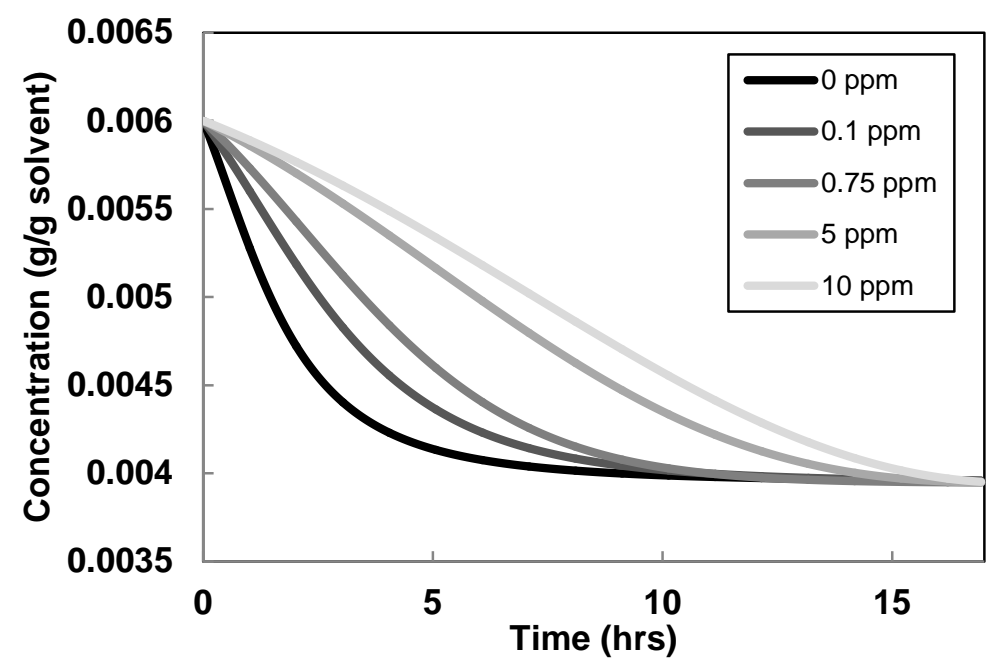

Figure 12: Simulated concentration profiles for seeded desupersaturation experiments of OABA at different concentrations of HPMC. 